\title{
Flavonóides glicosilados de Herissantia tiubae (K. Schum) Brizicky (Malvaceae) e testes farmacológicos preliminares do canferol 3,7-di-O- $\alpha$-L-ramnopiranosídeo
}

\author{
D.A. Silva ${ }^{1}$ D.A. Costa ${ }^{1}$, D.F. Silva ${ }^{1}$, M.F.V. Souza ${ }^{1 *}$, M.F. Agra ${ }^{1}$, I.A. Medeiros ${ }^{1}$, J.M. \\ Barbosa-Filho', R. Braz-Filho ${ }^{2}$
}

\author{
${ }^{1}$ Laboratório de Tecnologia Farmacêutica "Delby Fernandes de Medeiros", Universidade Federal da Paraíba, \\ Caixa Postal 5009, 58051-970, João Pessoa, PB, Brasil, \\ ${ }_{2}^{2}$ Setor de Química de Produtos Naturais, Universidade Estadual Norte Fluminense, \\ 28015-620, Campos, RJ, Brazil
}

\begin{abstract}
RESUMO: Das partes aéreas de Herissantia tiubae (K. Schum.) Brizicky foram isolados, através de métodos cromatográficos, dois flavonóides glicosilados, canferol 3,7-di-O- $\alpha$-L-ramnopiranosídeo e canferol 3- $\beta$-O-D-(6"-E- $p$-cumaroil) glicosídeo. As estruturas foram identificadas com o uso de técnicas espectroscópicas de IV, RMN ${ }^{1} \mathrm{H}$ e ${ }^{13} \mathrm{C}$ incluindo métodos bidimensionais, além de comparações com dados da literatura. O canferol 3,7-di-O- $\alpha$-L-ramnopiranosídeo foi submetido a testes farmacológicos preliminares com a finalidade de avaliar o seu efeito sobre o sistema cardiovascular.
\end{abstract}

Unitermos: Herissantia tiubae, Malvaceae, flavonóides, atividade cardiovascular.

\begin{abstract}
Glycosyl flavonoids from Herissantia tiubae (K. Schum) Brizicky (Malvaceae) and preliminary tests of kaempferol 3,7-di- $O$ - $\alpha$-L-rhamnopyranoside". From the aerial parts of Herissantia tiubae (K. Schum.) Brizicky two flavonol glycosides were isolated by means of chromatographic methods, kaempferol 3,7-di- $O$ - $\alpha$-L-rhamnopyranoside and kaempferol 3-O- $\beta$-D(6"'-E-p-coumaroyl) glucoside. Their structural identification was made by $\mathrm{IV},{ }^{1} \mathrm{H}$ and ${ }^{13} \mathrm{C}$ NMR spectroscopy, including two dimensional techniques, together with comparison with literatura data. Preliminary tests were carried out with kaempferol 3,7-di- $O$ - $\alpha$-L-rhamnopyranoside in order to study its possible cardiovascular effect.
\end{abstract}

Keywords: Herissantia tiubae, Malvaceae, flavonoids, cardiovascular activity.

\section{INTRODUÇ̃̃̃O}

A família Malvaceae é constituída por 243 gêneros e 4225 espécies (Stevens, 2003). Membros desta família ocorrem por quase todas as partes do mundo, com exceção de regiões muito frias, e são particularmente abundantes nas regiões tropicais, principalmente na América do Sul (Heywood, 1993). Muitas espécies da família Malvaceae são largamente usadas na terapêutica, como emolientes, anti-febris, diuréticos, antiinflamatórios e no tratamento de reumatismos, entre outras aplicações (Ahmed et al., 1990).

Herissantia tiubae é uma espécie restrita ao Nordeste do Brasil, onde ocorre em áreas do semiárido, no domínio da caatinga, sendo encontrada em grandes populações, em áreas abertas e ensolaradas, principalmente às margens das estradas (Agra, 1996).

$\mathrm{O}$ presente trabalho trata do isolamento e identificação de dois flavonóides, canferol 3,7-di-O$\alpha$-L-ramnopiranosídeo (1), também conhecido como lespedina e canferol 3-O- $\beta$-D-(6"-E- $p$-cumaroil) glicosídeo (2), também descrito como tilirosídeo, já relatados na literatura (Berrondo et al., 2003; Kaouadji, 1990), mas ainda não isolados no gênero Herissanitia, bem como testes preliminares com o canferol 3,7-di- $O$ $\alpha$-L-ramnopiranosídeo de maneira a avaliar sua atividade sobre o sistema cardiovascular.

\section{MATERIAL E MÉTODOS}

\section{Equipamentos}

Os dados espectrais de infravermelho foram obtidos em aparelho Perkin-Elmer, FT-IR-1750 utilizando-se de $3 \mathrm{mg}$ de amostra em pastilhas de $\mathrm{KBr}$, com freqüência medida $\mathrm{em} \mathrm{cm}^{-1}$. Os espectros de RMN foram obtidos em espectrômetro Mercury-Varian a $200\left({ }^{1} \mathrm{H}\right)$ e $50\left({ }^{13} \mathrm{C}\right)$, otimizados para técnicas uni e bidimensionais, sendo as amostras solubilizadas em $\mathrm{CD}_{3} \mathrm{OD}$. 


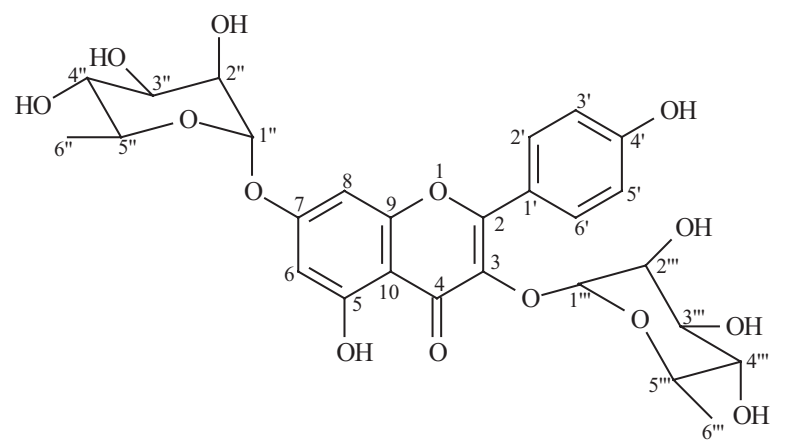

1<smiles>O=C(/C=C/c1ccc(O)cc1)OCC1C(O)OC(Oc2c(-c3ccc(O)cc3)oc3cc(O)cc(O)c3c2=O)C(O)C1O</smiles>

2

Figura 1. Substâncias isoladas de Herissantia tiubae

\section{Material botânico}

O material vegetal foi coletado em janeiro de 1994 na Região dos Cariris Velhos no município de Juazeirinho, Paraíba. A identificação da planta foi realizada pela botânica Prof ${ }^{a}$ Dra. Maria de Fátima Agra, do Núcleo de Pesquisas em Produtos Naturais/LTF/UFPB. Uma exsicata do material vegetal ( $\left.\mathrm{N}^{\circ} 2434\right)$ encontra-se arquivada no Herbário Prof. Lauro Pires Xavier (CCEN/ UFPB).

\section{Extração e isolamento dos constituintes químicos}

As partes aéreas de $H$. tiubae foram desidratadas em estufa em temperatura de $40{ }^{\circ} \mathrm{C}$ durante 96 horas e trituradas, sendo o pó $(5 \mathrm{Kg})$ macerado com etanol comercial a $95 \%$ por 72 horas, e extraído até exaustão. A soluçãoetanólica foiconcentradaemrotavapor, produzindo $300 \mathrm{~g}$ de extrato etanólico bruto, que foi solubilizado com solução etanol:água (7:3) e particionado com hexano, clorofórmio, acetato de etila e $n$-butanol. As fases acetato de etila e n-butanólica foram submetidas a sucessivas cromatografias em coluna, utilizando-se Sephadex LH20 como adsorvente e metanol como eluente. As frações obtidas foram comparadas por cromatografia em camada delgada de sílica gel, reunindo-se as semelhantes. Este procedimento resultou no isolamento do canferol 3,7-di$O$ - $\alpha$-L-ramnopiranosídeo ou lespedina $(30 \mathrm{mg})(\mathbf{1})$ da fase acetato de etila e do canferol 3-O- $\beta$-D-(6" $-E-p$-cumaroil) glicosídeo ou tilirosídeo $(50 \mathrm{mg})(2)$ da fase $n$-butanólica. Estas substâncias foram identificadas usando-se IV, RMN ${ }^{1} \mathrm{H}$ e ${ }^{13} \mathrm{C}$, incluindo técnicas bidimensionais (COSY, HETCOR e HMBC), bem como comparações com dados relatados na literatura.

\section{Dados espectroscópicos das substâncias isoladas}

Canferol 3,7-di-O- $\alpha$-L-ramnopiranosídeo ou lespedina (1): Cristais amarelos. RMN ${ }^{1} \mathrm{H}(200 \mathrm{MHz}$, CD 3 OD): 0,92 (3H, d, J 5,6 Hz, H-6" '); 1,26 (3H, d, J $6,0 \mathrm{~Hz}, \mathrm{H}-6 "$ ); 3,34 (1H, m, H-5"'); 3,47 (1H, t, J 9,4 Hz, H-4"'); 3,71 (1H, dd, J 9,4 e 3,4 Hz, H-3"' ); 3,83 (1H, $d$, $J$ 9,4 e 3,4 Hz, H-3"); 4,02 (1H, m, H-2"); 4,22 (1H, dd, $J$ 9,4 e 3,4 Hz, H-2"'); 5,38 (1H, dd, J 1,8 Hz, H-1"'); 5,55 (1H, sl, H-1"); 6,43 (1H, sl, H-6); 6,68 (1H, sl, H8); 6,92 (2H, $d, J 8,0 \mathrm{~Hz}, \mathrm{H}-3$ ', 5' ); 7,76 (2H, $d, J$ 8,0 Hz, H-2', 6').

Canferol 3-O- $\beta$-D-(6”-E-p-cumaroil) glicosideo ou tilirosídeo (2): Sólido amarelo. RMN ${ }^{1} \mathrm{H}(200 \mathrm{MHz}$, CD OD): 3, 16-3,25 (1H, $m, \mathrm{H}-5$ "'); 3,34-3,38 (3H, $m, \mathrm{H}-$ 2 ", H-3", H-4"); 4.06 (1H, dd, J 11,6 e 6,4 Hz, H-6"); 4.19 (dd, J = 11.8 e $2.2 \mathrm{~Hz}, \mathrm{H}-6 "$ ); 5,23 (1H, $d, J$ 7,6 Hz, $\mathrm{H}-1$ "); 6,05 (1H, d, J $16 \mathrm{~Hz}, \mathrm{H}-\alpha) ; 6,11(1 \mathrm{H}, d, J 2 \mathrm{~Hz}$, H-6); 6,27 (1H, d, J 2 Hz, H-8); 6,77 (2H, d, J 8,6 Hz, H-3"', ,5"'); 6,79 (2H, $d, J 9 \mathrm{~Hz}, \mathrm{H}-3$ ',5'); 7,25 (2H, $d, J$ $8,6 \mathrm{~Hz}, \mathrm{H}-2$ "', 6 "' ); 7,38 (1H, $d, J 16 \mathrm{~Hz}, \mathrm{H}-\beta) ; 7,96(2 \mathrm{H}$, d, J $9 \mathrm{~Hz}, \mathrm{H}-2$ ', 6').

\section{Preparações com artéria mesentérica superior isolada de rato com ou sem endotélio funcional}

Os ratos foram sacrificados por concussão cerebral seguida por secção dos vasos cervicais. Através de uma incisão no abdome do animal, retirou-se a artéria mesentérica superior. Anéis do primeiro segmento da artéria ( $1-2 \mathrm{~mm}$ ) foram obtidos livres de tecido conectivo 
Tabela 1. Dados de $\mathrm{RMN}{ }^{13} \mathrm{C}\left(\mathrm{CD}_{3} \mathrm{OD}, 50 \mathrm{MHz}\right)$ das substâncias 1 e canferol 3,7-di-O- $\alpha$-L-ramnosídeo $\left(\mathrm{CD}_{3} \mathrm{OD}, 50 \mathrm{MHz}\right)$

\begin{tabular}{|c|c|c|}
\hline $\mathrm{C}$ & $\begin{array}{c}1 \\
\delta_{\mathrm{C}} \\
\end{array}$ & $\begin{array}{c}\text { canferol 3,7-di-O- } \alpha \text {-L-ramnosídeo } \\
\delta_{\mathrm{C}} \\
\end{array}$ \\
\hline 2 & 158,0 & 159,2 \\
\hline 3 & 136,4 & 136,4 \\
\hline 4 & 179,7 & 179,7 \\
\hline 5 & 162,9 & 162,9 \\
\hline 6 & 99,8 & 99,8 \\
\hline 7 & 163,5 & 163,5 \\
\hline 8 & 95,6 & 95,5 \\
\hline 9 & 159,7 & 157,9 \\
\hline 10 & 107,5 & 107,5 \\
\hline 1 ' & 122,3 & 122,3 \\
\hline 2 ' & 132,0 & 131,9 \\
\hline $3^{\prime}$ & 116,5 & 116,6 \\
\hline 4 ' & 161,7 & 161,7 \\
\hline 5 , & 116,5 & 116,6 \\
\hline 6 & 132,0 & 131,9 \\
\hline 1, & 100,5 & 100,5 \\
\hline 2, & 71,7 & 72,1 \\
\hline $3^{\prime \prime}$ & 72,0 & 72,1 \\
\hline 4, & 73,8 & 73,1 \\
\hline $5^{\prime \prime}$ & 71,2 & 71,2 \\
\hline 6, & 18,0 & 18,1 \\
\hline 1, & 103,5 & 103,5 \\
\hline 2, & 71,9 & 71,8 \\
\hline
\end{tabular}

e adiposo. Os anéis foram mantidos em cubas contendo $10 \mathrm{~mL}$ de solução de Tyrode, a $37^{\circ} \mathrm{C}$ e gaseificada com uma mistura de $95 \%$ de $\mathrm{O}_{2}$ e $5 \%$ de $\mathrm{CO}_{2}$ (carbogênio). Os anéis foram suspensos por linhas de algodão fixadas a um transdutor de força acoplado a um sistema de aquisição (Miobath-4, WPI, Sarasota, EUA) para o registro das tensões isométricas. Cada anel foi submetido a uma tensão constante de $0,75 \mathrm{~g}$ por um período de 60 min. Durante este tempo, o meio nutritivo foi trocado a cada $15 \mathrm{~min}$. para prevenir a interferência de metabólitos (Altura; Altura, 1970).

Os anéis sem endotélio foram obtidos mecanicamente através do atrito entre as paredes internas do vaso com o auxílio de um "swab" de algodão.

Nos protocolos experimentais com ou sem endotélio funcional, após um período de estabilização de 60 minutos, foram induzidas três contrações similares com $10 \mu \mathrm{M}$ de fenilefrina e após a estabilização da terceira contração (20 min), concentrações crescentes de canferol 3,7-di- $O$ - $\alpha$-L-ramnopiranosídeo foram adicionadas à cuba de maneira cumulativa na fase tônica da terceira contração, sendo observado efeito sobre a contração induzida por fenilefrina.

Esta pesquisa foi aprovada pelo Comitê de Ética em Pesquisa da Universidade Federal da Paraíba, através do parecer CEP/LTF/UFPB n ${ }^{\circ}$ 01/03.

\section{RESULTADOS E DISCUSSÃO}

O espectro de infravermelho de 1 (Figura 1) mostrou uma banda larga em $3374 \mathrm{~cm}^{-1}$, sugerindo grupo $\mathrm{OH}$ fenólico e/ou de álcool. Esta absorção foi corroborada pelo pico em $1212 \mathrm{~cm}^{-1}$, típico de deformação axial de C-O. Absorções para deformação axial de $\mathrm{C}=\mathrm{C}$ de aromáticos foram observadas entre 1602 e $1449 \mathrm{~cm}^{-1}$. Duas bandas em 2981 e 2937 cm$^{-1}$ referem-se ao estiramento C-H de grupos metílicos, metínicos e/ou metilênicos. Uma forte absorção em $1659 \mathrm{~cm}^{-1}$ permitiu inferir sobre a presença de uma carbonila conjugada ou formando ponte de hidrogênio. Comparando-se estes valores com espectros de IV de flavonóides já descritos (Costa, 2002), pôde-se sugerir que 1 tratava-se de uma substância desta classe.

$\mathrm{O}$ espectro de $\mathrm{RMN}{ }^{1} \mathrm{H}$ da substância 1 exibiu dois dubletos com sinais intensos em $\delta 8,06$ e $\delta 6,88$, integrando para dois hidrogênios cada, o que permitiu sugerir um sistema AA'BB'. Singletos largos em $\delta 6,38 \mathrm{e}$ $\delta$ 6,70 mostraram tratar-se das absorções dos hidrogênios 6 e 8 do anel A do esqueleto de flavonóides 5,7diidroxilados (Harborne, 1994). A proposta da substância 1 possuir um esqueleto do tipo flavonol foi corroborada pela ausência, no espectro de $\mathrm{RMN}^{1} \mathrm{H}$, de um singleto com absorção entre $\delta 6,39$ e $\delta 6,94$, o qual seria característico do hidrogênio 3 das 5,7-diidroxiflavonas (Harborne, 1994). Adicionalmente neste espectro, foram reveladas 
absorções que permitiram inferir sobre a presença de duas unidades de açúcar. Estas absorções correspondiam a multipletos entre $\delta 3,30$ e $\delta 4,02$ e dois sinais em $\delta 5,55$ e $\delta 5,38$, correspondentes a hidrogênios anoméricos e dois dubletos adicionais em $\delta 1,26$ e $\delta 0,92$, referentes a hidrogênios metílicos de ramnose, (Rasoanaivo et al, 1990).

Segundo Harborne (1994), quando a ramnose ocorre na posição 3 em esqueleto do tipo canferol, o valor de deslocamento químico dos hidrogênios metílicos e anomérico ficam entre $\delta$ 0,72-0.86 e $\delta$ 4,96-5,36, respectivamente, enquanto que a ramnose na posição 7 mostra tais sinais entre $\delta 1,04-1,21$ e $\delta$ 5,22-5,75 para os referidos hidrogênios. Como foram observadas as absorções em $\delta 1,25$ para os hidrogênios metílicos e $\delta$ 5,55 para o hidrogênio anomérico de uma das unidades de ramnose e $\delta 0,92\left(\mathrm{CH}_{3}-6\right.$ ") e $\delta 5,38(\mathrm{H}-1$ ") para a outra unidade, foi possível então sugerir que as glicosilações ocorreriam nos carbonos 7 e 3 . O espectro de $\mathrm{RMN}{ }^{13} \mathrm{C}$ corroborou com a sugestão da presença de duas ramnoses ao revelar sinais para dois carbonos anoméricos em $\delta$ 100,57 e $\delta 103,50$ e ainda para os dois carbonos metílicos em $\delta 18,09$ e $\delta 17,66$, além dos demais picos da ramnose entre $\delta 71,28$ e $\delta 73,86$.

$\mathrm{O}$ espectro de $\mathrm{RMN}{ }^{1} \mathrm{H} \times{ }^{1} \mathrm{H}-\mathrm{COSY}$ favoreceu as atribuições dos hidrogênios do sistema AA'BB' do canferol, bem como das moléculas de ramnose através do acoplamento seqüenciado dos hidrogênios destes açúcares pelas interações seguintes: $\mathrm{H}-2^{\prime} / 6^{\prime}$ com $\mathrm{H}-$ 3'/5' (anel B); H-2"/H-3"; H-3"/H-4"; H-4"/H-5"; H-5"/H-6"; H-1"'/H-2",; H-2""/H-3" ; H-3" /H-4",; $\mathrm{H}-4$ "'/H-5",; H-5"'/H-6",. Os carbonos respectivos dos hidrogênios das unidades de ramnose foram identificados pelas correlações ${ }^{1} \mathrm{~J} \mathrm{C}$-H com base no espectro de RMN ${ }^{1} \mathrm{H} \mathrm{x}{ }^{13} \mathrm{C}$-HMQC.

O espectro de HMBC mostrou a correlação a três ligações entre o hidrogênio anomérico em $\delta$ 5,55 (H-1") com o carbono 7 ( $\delta 163,50)$, bem como entre o hidrogênio em $\delta 5,38$ (H-1 "') com o carbono 3 ( $\delta 136,47)$, estabelecendo assim as posições das unidades de ramnose nos carbonos 7 e 3 respectivamente. As atribuições de hidrogênios e carbonos do anel B foram feitas com base nas correlações seguintes: C-2 ( $\delta$ 158,01) com H-2'/H-6' $\left({ }^{3} J\right)$; C-2' $(\delta 122,38)$ com H-3'/H-5' ( $\left.{ }^{3} J\right)$ e C-4' $(\delta 161,72)$ com H-2'/H-4'. As correlações do H-8 $(\delta 6,68)$ a duas ligações com C-9 $(\delta 159,75)$ e a três ligações com C-6 $(\delta$

Tabela 2. Dados de $\mathrm{RMN}{ }^{1} \mathrm{H}$ e ${ }^{19} \mathrm{C}\left(\mathrm{CD}_{3} \mathrm{OD}, 50 \mathrm{MHz}\right)$ das substâncias 2 e canferol-3-O- $\beta$-D-(6' $-E-p$-cuma glicosídeo $\left(\mathrm{CD}_{3} \mathrm{OD}, 75 \mathrm{~Hz}\right)$

\begin{tabular}{|c|c|c|}
\hline $\mathrm{C}$ & $\begin{array}{c}2 \\
\delta_{\mathrm{C}} \\
\end{array}$ & $\begin{array}{c}\text { canferol-3-O- } \beta \text {-D-(6" }-E-p \text {-cumaroil) glicosídeo } \\
\delta_{\mathrm{C}}\end{array}$ \\
\hline 2 & 159,2 & 159,2 \\
\hline 3 & 135,2 & 135,2 \\
\hline 4 & 179,3 & 179,2 \\
\hline 5 & 162,8 & 162,7 \\
\hline 6 & 99,9 & 99,9 \\
\hline 7 & 165,7 & 165,7 \\
\hline 8 & 94,8 & 94,9 \\
\hline 9 & 158,2 & 158,2 \\
\hline 10 & 105,5 & 105,0 \\
\hline 1 ' & 122,6 & 122,6 \\
\hline 2 ' & 132,1 & 132,2 \\
\hline 3 , & 115,9 & 116,7 \\
\hline 4 , & 161,4 & 161,4 \\
\hline 5 & 115,9 & 116,7 \\
\hline 6 ' & 132,1 & 132,2 \\
\hline 1, & 104,0 & 104,1 \\
\hline $2^{\prime \prime}$ & 75,7 & 75,7 \\
\hline 3, & 77,9 & 78,0 \\
\hline 4, & 71,6 & 71,7 \\
\hline 5, & 75,7 & 75,7 \\
\hline 6, & 64,3 & 64,3 \\
\hline $1 \%$, & 127,0 & 127,0 \\
\hline $2^{\prime \prime \prime}$ & 131,1 & 131,1 \\
\hline 3, & 116,7 & 116,0 \\
\hline $4^{\prime \prime}$, & 161,0 & 161,0 \\
\hline 5, & 116,7 & 116,0 \\
\hline $6 \%$ & 131,1 & 131,1 \\
\hline$\alpha$ & 114,7 & 114,7 \\
\hline$\beta$ & 146,5 & 146,5 \\
\hline
\end{tabular}


$99,86)$ e $\mathrm{C}-10(\delta 107,53)$ favoreceram os assinalamentos pertinentes ao anel A do esqueleto do canferol A análise dos dados espectrais, bem como as comparações com a literatura (Berrondo et al., 2003) permitiram atribuir a estrutura do canferol 3,7-di-O- $\alpha$-L-ramnopiranosídeo para a substância 1 (Tabela 1).

Pela análise do espectro de IV de 2 (Figura 1) observaram-se duas bandas de absorção em 3460 e 3276 $\mathrm{cm}^{-1}$, típicas de deformação axial de $\mathrm{O}-\mathrm{H}$ em ligação hidrogênio intermolecular (Silverstein; Bassler, 1994). Uma absorção adicional em $1363 \mathrm{~cm}^{-1}$, referente à deformação angular no plano de O-H, corroborou com a presença de hidroxila de fenol e/ou álcool na molécula. A existência de anel aromático pôde ser inferida a partir de um conjunto de bandas observado entre 1607 e 1501 $\mathrm{cm}^{-1}$, referentes à deformação axial de $\mathrm{C}=\mathrm{C}$ de aromático. Uma banda em $1655 \mathrm{~cm}^{-1}$ sugeriu a presença de uma carbonila em ponte na molécula de $\mathbf{2}$, condizente com a absorção atribuída para deformação axial de $\mathrm{O}-\mathrm{H}$ em ligação hidrogênio intermolecular referida acima. Tais absorções são comuns em espectros de IV de alguns flavonóides (Costa, 2002). Uma banda intensa em 1684 $\mathrm{cm}^{-1}$ é típica de uma função carbonila conjugada de éster (Pavia et al., 1996). Esta proposta encontrou suporte nas bandas entre 1297 e $1068 \mathrm{~cm}^{-1}$, atribuídas a estiramento C-O de ésteres. (Pavia et al, 1996).

Os espectros de $\mathrm{RMN}{ }^{1} \mathrm{H}$ exibiram um conjunto de absorções na região de hidrogênios aromáticos que permitiu inferir sobre um esqueleto típico de flavonóide 5,7-dioxigenado (Harborne, 1994). Um dubleto em $\delta$ 6,11 e outro em $\delta 6,27$, ambos com uma constante de acoplamento $(J)$ igual a $2,0 \mathrm{~Hz}$, mostrando acoplamento meta, são comuns aos hidrogênios 6 e 8 do esqueleto flavonoídico substituído nas posições 5 e 7 do anel A do canferol, fortalecendo o que foi inferido sobre um esqueleto do tipo 5,7-dioxigenado. Dois dubletos em $\delta 7,96$ e $\delta 6,79$, integrando para $2 \mathrm{H}$ cada e $J=9,0 \mathrm{~Hz}$, característico de acoplamento orto, demonstraram a presença de um sistema AA'BB', sugerindo assim um esqueleto flavonoídico com anel B para substituído para a substância 2. A ausência de um singleto na região entre $\delta$ 6,39 e $\delta$ 6,94 no espectro de $\mathrm{RMN}{ }^{1} \mathrm{H}$ sugeriu que a posição C-3 do anel C em 2 encontrava-se substituída. O espectro mostrou ainda dois pares de dubletos adicionais, sendo um deles em $\delta 6,77$ e $\delta 7,25(J=8,6 \mathrm{~Hz})$ referente a outro sistema AA'BB' e outro par em $\delta 6,05$ e $\delta 7,38$, com uma constante de acoplamento de $16 \mathrm{~Hz}$, sugerindo uma unidade de ácido $p$-cumárico com configuração trans $(E)$ (Nasr et al., 1987; Brasseur; Angenot, 1988). A presença de uma unidade osídica fazendo parte da molécula pôde ser sugerida pelo dubleto em $\delta 5,23 \mathrm{com} J=7,6 \mathrm{~Hz}$, típico de hidrogênio anomérico de glicose com configuração $\beta$ (Kaouadji, 1990). Esta sugestão pôde ser reforçada pelas absorções em $\delta 4,19$ e $\delta 4,06$, referentes a dois duplo dubletos, normalmente atribuídos aos hidrogênios metilênicos do carbono 6 na molécula da glicose (Fico et al., 2001). As evidências para um flavonóide 5,7dioxigenado e a presença do sistema AA'BB', bem como a absorção ausente para o hidrogênio 3 , comum às 5,7diidroxiflavonas, sugeriram que a substância 2 tratava-se de um flavonóide formado por um padrão de substituição do tipo do canferol (3,5,7-4'-tetrahidroxiflavonol), contendo uma unidade de açúcar e outra de ácido $p$ cumárico.

Através da análise do espectro de $\mathrm{RMN}{ }^{13} \mathrm{C}$, observaram-se absorções para 30 átomos de carbono. Deslocamentos químicos para o padrão do canferol (3,5,7,4'-tetrahidroxiflavona) puderam ser distintos pelos

\section{canferol \\ 3,7-di-O- $\alpha$-L-ramnopiranosídeo}

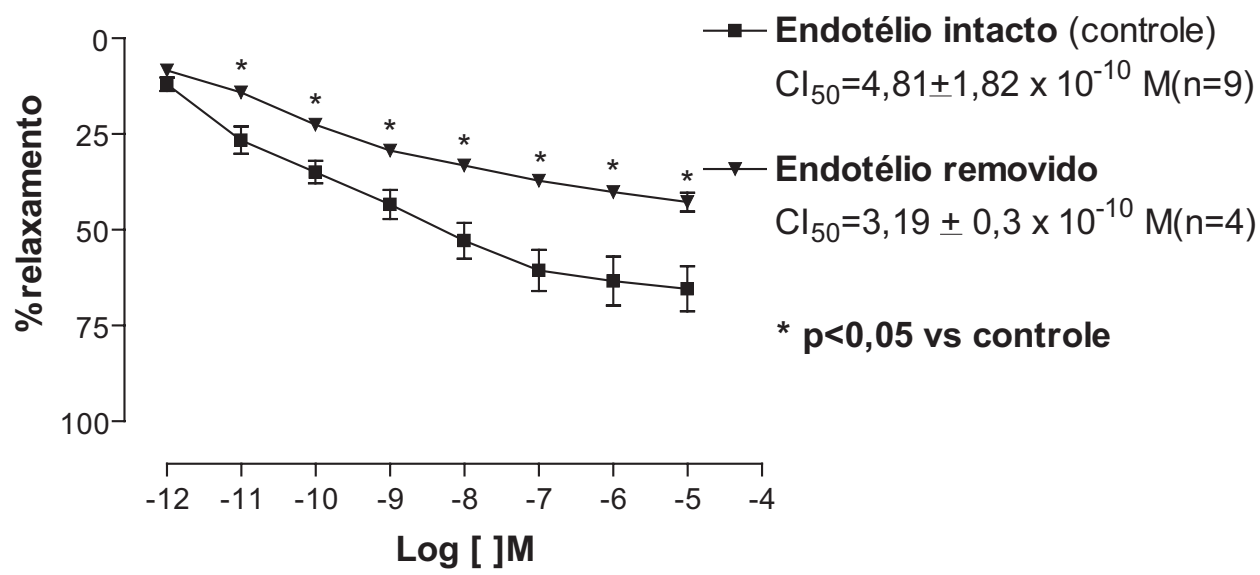

Figura 2. Curva concentração-resposta do canferol 3,7-di- $O$ - $\alpha$-L-ramnopiranosídeo $\left(10^{-12}\right.$, $10^{-11}, 10^{-10}, 10^{-9}, 10^{-8}, 10^{-7}, 10^{-6}$ e $\left.10^{-5} \mathrm{M}\right)$ em anéis de artéria mesentérica superior isolada de rato pré-contraídas com fenilefrina $(10 \mu \mathrm{M})$ na presença e na ausência de endotélio vascular. Os valores são expressos como média \pm EPM. 
picos em $\delta 179,30$, referente a grupo carbonila proposto pela absorção em $1655 \mathrm{~cm}^{-1}$ no IV, com substituintes oxigenados nas posições 3 e 5 (Agrawal, 1989), bem como em $\delta 135,22, \delta 162,82, \delta 165,79$ e $\delta 161,43$, condizentes com os carbonos não hidrogenados 3,5 , 7 e 4', respectivamente, segundo comparações com a literatura (Carlton et al., 1990). Um pico em $\delta 168,82$, típico de carbonila $\alpha, \beta$-insaturada (Carlton et. al., 1990), reiterou as sugestões a respeito de uma unidade de ácido $p$-cumárico, feitas pelo IV $\left(1684 \mathrm{~cm}^{-1}\right)$ para carbonila de éster e pelo RMN ${ }^{1} \mathrm{H}$ quando se referiu a um par de dubletos em $\delta 6,05$ e $\delta 7,38$ com constantes de acoplamento de $16 \mathrm{~Hz}$, indicando configuração trans. A proposta para a presença de uma molécula de glicose foi corroborada pela absorção em $\delta 104,02$, que se refere a carbono anomérico. A unidade de ácido $p$-cumárico foi sugerida estar ligada ao carbono 6" da glicose. Tal sugestão foi suportada pelo fato de que este carbono quando livre, absorve normalmente em $\delta 62,0$, sendo este valor levado a campo baixo pela porção cumaroil (Kaouadji, 1990).

O espectro de RMN ${ }^{13} \mathrm{C}$ mostrou também quatro sinais intensos referentes a dois sistemas AA'BB'. As duas absorções em $\delta 132,19$ e $\delta 115,99$ foram propostas para os carbonos 2',6' e 3', 5', respectivamente, do anel B do flavonóide. Esta proposta foi confirmada pela correlação a três ligações entre os hidrogênios 2', 6' com o carbono 2 através do espectro de HMBC e conseqüentemente pela correlação direta destes hidrogênios com os carbonos 2',6' por meio do espectro de HETCOR. Os dados de HMBC confirmaram os deslocamentos químicos de carbonos e hidrogênios para a unidade cumaroil ao mostrar as seguintes correlações: $\delta 168,82$ (O) com o hidrogênio $\beta(\delta 7,38)$ a três ligações; $C-1$ " " $(\delta 127,03)$ com os hidrogênios H- $\alpha(\delta 6,05)$ e H-3" "/5" ' $(\delta 6,77)$ a três ligações e C-4"' $(\delta 161,07)$ a ${ }^{3} J$ com os hidrogênios $2 " \prime / 6 ",(\delta 7,25)$. Os sistemas AA'BB' foram também confirmados pelos acoplamentos mostrados pelo espectro de ${ }^{1} \mathrm{H} \mathrm{x}{ }^{1} \mathrm{H}-\mathrm{COSY}$, que revelou a acoplamento entre os pares de hidrogênios H-2', 6' com H-3', 5' e H-2", 6' com H-3",6". O acoplamento seqüenciado entre os hidrogênios H-1"'/H-2"'; H-2"'/H-3"'; H-3"'/H-4"'; H-4"'/H-5" e $\mathrm{H}-5$ "'/H-6" permitiram atribuir os assinalamentos da molécula de glicose, cujos carbonos respectivos foram identificados pelas interações ${ }^{1} J$ exibidas pelo espectro de HETCOR. O dados espectrais da substância 2 foram também comparados com a literatura (Kaouadji, 1990) permitindo identificá-la como sendo o canferol-3-O- $\beta$-D(6"-E-p-cumaroil) glicosídeo (Tabela 2) .

Observou-se que o canferol 3,7-di-O- $\alpha$-Lramnopiranosídeo nas concentrações de $10^{-12}, 10^{-11}$, $10^{-10}, 10^{-9}, 10^{-8}, 10^{-7}, 10^{-6}$ e $10^{-5} \mathrm{M}$ promoveu um efeito vasorelaxante em anéis de artéria mesentérica superior isolada de rato, pré-contraídas com fenilefrina $10 \mu \mathrm{M}$, de maneira concentração dependente, apresentando um efeito máximo em torno de $65,48 \pm 5,86 \%$ (Figura 2). Este efeito foi atenuado significantemente em relação aos experimentos com anéis de artéria mesentérica sem endotélio funcional, com diminuição da resposta máxima (Emax $=42,83 \pm 2,45 \%, n=4)$. No entanto, a potência farmacológica não apresentou diferenças significativas entre os anéis com endotélio intacto ou endotélio removido. Estes resultados são extremamente importantes no âmbito da farmacologia cardiovascular, visto que, artérias de resistência, como a artéria mesentérica (Horie et al, 2003), estão totalmente implicadas no controle da pressão arterial, a qual corresponde ao produto do débito cardíaco (DC) pela resistência vascular periférica total (RVPT) (Oates, 1996). Baseando-se nisto, drogas que tenham propriedades vasorelaxantes sobre as referidas artérias, podem ser, após estudos aprofundados, futuros agentes anti-hipertensivos ou novas ferramentas farmacológicas de estudo, contribuindo desta forma para a terapêutica contra um dos principais problemas de Saúde Pública, a hipertensão, que trata-se de uma condição clínica na qual a pressão do sangue está acima das medidas consideradas normais por um período longo de tempo, sendo uma conseqüência de distúrbios na regulação da pressão arterial e uma das principais causas de doenças cardiovasculares. Este distúrbio tem demonstrado uma ocorrência elevada na população brasileira. Em média, a prevalência se situa em cerca de $15 \%$ da população geral adulta, variando conforme o estudo e a localidade pesquisada de 9 a 30\% (Kaplan, 2001).

Os nossos estudos com canferol 3,7-di- $O-\alpha-\mathrm{L}-$ ramnopiranosídeo são preliminares, mas suficientes para serem levados adiante.

\section{AGRADECIMENTOS}

Os autores agradecem ao CNPq pelo auxílio financeiro. A Vicente Carlos da Costa pela obtenção dos espectros RMN. Ao Banco de Dados NAPRALERT pelo levantamento bibliográfico do gênero Herissantia.

\section{REFERÊNCIAS}

Agra MF 1996. Plantas da medicina popular dos Cariris Velhos, Paraíba. João Pessoa: Editora União.

Agrawal PK 1989. Carbon-13 NMR of flavonoids: Studies in organic chemistry 39. India: Elsevier.

Ahmed Z, Kazmi SNH, Walik 1990. A Phytochemical investigation of Abutilon pakistanicum. J Nat Prod 53: $1342-1344$

Altura BM, Altura BT 1970. Differential effects of substrate depletion on drug-induced contractions of rabbit aorta. Am J Physiol 219: 1698-1705.

Berrondo LF, Gabriel FT, Fernandes SBO, Menezes FS 2003. Dirhamnosyl flavonoids and other constituents from Brillantaisia palisatii. Quím Nova 26: 922-923.

Brasseur T, Angelot L 1988. Six flavonol glycosides from leaves of Strychnos variabilis. Phytochemistry 27: 1487-1490.

Carlton RR, Gray AI, Lavaud C, Massiot G, Waterman PG 1990. Kaempferol-3-(2,3-Diacetoxy-4-paracoumaroyl)-rhamnoside from leaves of Myrica gale. 
Phytochemistry 29: 2369-2371.

Costa DA 2002. Flavonas isoladas e identificadas das partes aéreas de Herissantia tiubae (K. Schum) Brizicky (Malvaceae). João Pessoa, 104 p. Dissertação de Mestrado - Laboratório de Tecnologia Farmacêutica, Universidade Federal da Paraíba.

Fico G, Braca A, De Tommasi N, Tomè F, Morelli I 2001. Flavonoids from Aconitum napellus subsp. Neomontanum. Phytochemistry 57: 543-546.

Harborne JB 1994. The flavonoids - advances in research since 1986. London: Chapman \& Hall.

Heywood VH 1993. Flowering plants of world. B. T. London: Batsford Ltd.

Horie T, Ohtsuru Y, Shibata K, Yamashiata K, Tsukayama M, Irvine JC, Favaloro JL, Kemp-Harper BK 2003. NO activates soluble guanylate cyclase and $\mathrm{Kv}$ channels to vasodilate resistence arteries. Hypertension 41: 1301-1307.

Kaouadji M 1990. Acylated and non-acylated kaempferol monoglycosides from Platanus acerifolia buds. Phytochemistry 29: 2295-2297.

Kaplan NM 2001. Systemic hypertension: mechanisms and diagnostics. In Braunwald Libby: Sauderes.

Nasr C, Guth AL, Berrurier MH, Anton R 1987. Quercetin coumaroyl glucorhamnoside from Ginkgo biloba. Phytochemistry 26: 2869-2870.

Oates JA 1996. Antihypertensive agents and the drug therapy of hypertension. In: Hardman JG, Gilman AG, Limbrind LE Goodman \& Gilman's. The pharmacolgogical basis of therapeutics. $9^{\mathrm{a}}$ ed. USA: McGraw-Hill.

Pavia DL, Lampman Kriz GS 1996. Introduction to spectroscopy - A guide for students of organic chemistry. $2^{\mathrm{a}}$ ed. USA: Saunders Golden Publishing.

Rasoanaivo P, Ratsimamangaurverg S, Messana I, Devicente Y, Galeffi C 1990. Cassinopin, a kaempferol trirhamnoside from Cassinopsis madagascariensis. Phytochemistry 29: 2040-2043.

Silverstein RM, Bassler GC 1994. Identificação espectrométrica de compostos orgâncos. $5^{\mathrm{a}}$ ed. São Paulo: Editora Guanabara Koogan S.A.

Stevens PF 2003. Angiosperm phylogeny website. http://www. mobot.org. Acessado em maio de 2003. 